\title{
Cyberbullying en Educación Primaria: Factores explicativos relacionados con los distintos roles de implicación
}

\author{
Cristina María GARCÍA-FERNÁNDEZ, Eva Ma ROMERA-FÉLIX \\ y Rosario ORTEGA-RUIZ \\ Universidad de Córdoba, España
}

(Recibido 27 Enero, 2017; Aceptado 16 Marzo, 2017)

\begin{abstract}
RESUMEN: El cyberbullying ha sido identificado como uno de los problemas más importantes entre los jóvenes en la última década. Las contribuciones científicas a este fenómeno se centran de forma destacada en el estudio de los factores asociados con los rasgos de personalidad, sobre todo en la etapa secundaria. El objetivo de este estudio es determinar si, junto con ciertas variables de personalidad existen variables del contexto que pueden explicar la participación en el acoso cibernético como no implicados, espectadores, víctimas, agresores y agresores victimizados. Un total de 1278 escolares de primaria (47.7\% niñas), con edades comprendidas entre los 10-14 años, en Andalucía $(M=11.11 ; D T=0.75)$ participaron en el estudio. Los análisis estadísticos indican que las variables tanto personales como interpersonales influyen en el acoso cibernético, específicamente, se observó el género, la autoestima negativa, el ajuste social entre iguales, y la disruptividad y conflictividad. Los resultados se discuten en función de las contribuciones científicas que explican la influencia de estos factores en los roles de participación en el acoso cibernético.

Palabras clave: cyberbullying; roles de participación; factores personales; factores del contexto escolar

\section{Cyberbullying in Primary School: Explicative Factors Related to Roles of Involvement}

\begin{abstract}
Cyberbullying has been identified as one of the most important problems among young people in the last decade. The scientific contributions to this phenomenon focus prominently on the study of factors associated with personality traits, mainly in the secondary stage. The aim of this study is to determine if together with certain personality variables exist school context variables that may explain involvement in cyberbullying as not involved, bystanders, victims, aggressors and aggressors victimized. A number of 1278 schoolchildren (47.7\% girls), of primary education aged 10-14 years, in Andalucía ( $M$ $=11.11 ; S D=0.75)$ participated in the study. The statistical analysis indicates that both personal and interpersonal variables are influential in cyberbullying, specifically it noted gender, negative self-esteem, social adjustment between peers, and disruption and conflict. The results are discussed on the basis of the scientific contributions that explain the influence of these factors in the roles of involvement in cyberbullying
\end{abstract}

Key words: cyberbullying; roles of involvement; personal factors; school context factor.

Correspondencia: Cristina María García-Fernández. Departamento de Psicología (Universidad de Córdoba), Avd. San Alberto Magno, s/n 14071 Córdoba. Teléfono/fax: 34957212513; e-mail: m12gafec@uco.es 
La vertiginosa ramificación del fenómeno bullying en otros tipos de violencia como es el caso del cyberbullying ha marcado una realidad social, que en los últimos años está teniendo un papel destacado entre los escolares (Garaigordobil, 2011; Olweus y Limber, 2010; Tokunaga, 2010). No obstante, la mayoría de los estudios realizados hasta el momento en nuestro país se centran principalmente en las etapas de educación secundaria (Álvarez-García et al., 2011; Avilés, 2009; Ortega, Calmaestra y Mora-Merchán, 2008; Sureda et al., 2009), tal vez debido a que el acceso a las tecnologías de la información y comunicación (en adelante TIC) en los escolares de primaria está menos presente (INE, 2012).

La literatura científica se ha centrado en el estudio de los factores de riesgo y de protección de las conductas de implicación, centrándose en el estudio de variables personales e interpersonales para llegar a describir los comportamientos de los escolares en el ciberacoso (Estévez, Villardón, Calvete, Padilla y Orue, 2010; Fanti, Demetruiou y Hawa, 2012; Hinduja y Patchin, 2013; Salmivalli y Pöyhönen, 2012).

En referencia a los factores de carácter personal, la edad y el género han sido señalados como unos de los primeros indicadores relacionados con el ciberacoso, aunque los diferentes estudios apuntan a resultados en algunas ocasiones contradictorios. Así, la edad ha sido enmarcada en torno a los 13-15 años señalándose en estas edades una mayor prevalencia (Garaigordobil, 2011; Tsitsika, et al., 2015; Tokunaga, 2010), aunque otros estudios identifican las etapas de primaria con una mayor implicación de chicos y chicas en comportamientos de cibervictimización y de ciberagresión (Dehue, Bolman y Vollink, 2008). En esta línea de investigación, también la literatura científica ha descrito que los estudiantes de los grados siete y ocho de EE.UU (equivalentes al primer ciclo de la ESO de España) desarrollan más conductas de cibervictimización, produciéndose un descenso en la etapas educativas posteriores (Schneider, O'Donnell, Stueve y Coulter, 2012; Tokunaga, 2010). Por su parte, Garaigordobil (2015) subraya una mayor incidencia de agresores y espectadores a medida que aumenta la edad (Garaigordobil, 2015), y otros estudios por el contrario no encuentran relación alguna (Hinduja y Patchin, 2008; Juvonen y Gross, 2008; Smith et al., 2008).

Los estudios relativos al género muestran diferencias en función del rol desempeñado en la dinámica, señalando una mayor implicación de los chicos como ciberagresores (Avilés, 2010; Calvete, Orue, Estévez, Villardón y Padilla, 2010; Erdur-Baker y Tanrikulu, 2010; Fanti, Demetruiou y Hawa, 2012; Salmivalli y Pöyhönen, 2012) y de las chicas como cibervíctimas (Calmaestra, Ortega y Mora-Merchán, 2008; Dehue et al., 2008; Estévez et al., 2010; Rivers y Noret, 2010).

La autoestima y el rendimiento académico también han sido objeto de estudio para la comprensión de las conductas de ciberacoso. Así, la baja autoestima ha sido reconocida como un factor clave, que podría constituirse no solo como la causa sino también como la consecuencia de los comportamientos de cibervictimización y ciberagresión (Estévez, et al., 2010; Tokunaga, 2010; Varjas, Henrich y Meyers, 2010). Los estudios reconocen que los agresores de cyberbullying podrían distanciarse física y psicológicamente del acto agresivo y, por consiguiente, de sus consecuencias sociales y psicológicas, por lo que su impacto sobre la autoestima podría verse disminuido (Kiriakidis y Kavoura, 2010). En cuanto a las cibervíctimas, presentan una baja autoestima (Cenat et al., 2014), aunque otros estudios han asociado esta baja autoestima tanto al grupo de los ciberagresores como al de las cibervíctimas (Brewer y Kerslake, 2015; Kowalski y Limber, 2013). 
En cuanto al rendimiento académico, niveles bajos de este factor en los implicados en ciberacoso han sido identificados como una consecuencia (Li, Smith y Cross, 2012; Tokunaga, 2010). De hecho, se ha reconocido en las víctimas un bajo rendimiento académico (Kowalski, Limber y Agatston, 2010; Manke, 2005). Sin embargo, otros estudios han caracterizado a las cibervíctimas como personas académicamente exitosas, identificando además a los chicos como el grupo que más desarrollara este rol (Avilés, 2010). Pero también, los estudios han señalado que el bajo rendimiento académico estaría relacionado con los ciberagresores y ciberagresores victimizados, siendo los chicos los que más dificultades presentaran (Avilés, 2010).

La asunción del cyberbullying como un fenómeno complejo y de carácter psicosocial ha alentado a los investigadores a estudiar otros factores de riesgo relativos a las características contextuales como son el ajuste social con los iguales y el ajuste a las normas sociales. Estos factores destacan la influencia de los iguales en el desarrollo de actitudes y comportamientos hacia la implicación en violencia (Steinberg y Monahan, 2007).

En un estudio realizado por Ortega, Del Rey y Sánchez (2011) se recoge un elevado número de contactos entre los jóvenes, lo que demuestra esa necesidad de comunicación y de vinculación dentro de las comunidades sociales que se establecen en el terreno virtual, revelándose esencial la inclusión en el grupo y el interés relacional de los escolares (Bringué y Sádaba, 2011; Kowalski, Limber y Agatston, 2008; Ortega et al., 2011).

El apoyo o rechazo social ha sido examinado como moderador en la relación entre la cibervictimización y el comportamiento ciberagresivo (Hanish y Guerra, 2000). En esta línea, existen estudios que sugieren que los escolares rechazados utilizan estrategias agresivas al interactuar con sus compañeros (Calvete et al., 2010; London, Downey, Bonica y Paltin, 2007), lo que les convierte en escolares con un bajo apoyo social (Calvete et al., 2010), y permite identificar a este factor como predictivo del comportamiento agresivo (Fite, Colder, Lochman y Wells, 2007; London et al., 2007). A pesar de ello, los agresores también son reconocidos como hábiles, populares y con cierto éxito social lo que les ayudaría a perpetrar sus actuaciones (Gini, Pozzoli y Hauser, 2011). A las víctimas se les reconoce un escaso apoyo social señalándolas como escolares con pocos amigos (Navarro, Yubero y Larrañaga, 2015), aunque también existen estudios que señalan que no existiría ninguna relación entre el apoyo social y la cibervictimización (Mishna et al., 2016).

En cuanto al ajuste a las normas sociales, autores como Scholte y sus colaboradores (2012) hallaron que a nivel de clase, las actitudes anti-bullying del alumnado se relacionaban negativamente con las conductas de ciberacoso.

El hecho de que el cyberbullying pueda darse fuera del espacio y horario escolar no lo convierte en una dinámica ajena a las normas de aula, así lo demuestran los estudios que describen cómo estas parecen tener cierta influencia en la aparición y desarrollo de las conductas de ciberacoso (Patchin y Hinduja, 2006; Shariff y Hoff, 2007). En esta línea, el trabajo de Hinduja y Patchin (2013) subraya que la influencia de las normas de convivencia en el aula pueden trascender del contexto escolar, siendo que cuando determinadas conductas son catalogadas como no apropiadas en ese contexto, los jóvenes son menos propensos a desarrollarlas en el escenario virtual.

La literatura sobre el ciberacoso subraya la influencia de características personales y las normas de contexto de los iguales sobre los comportamientos de ciberagresores y cibervictimas (Felst y Quandt, 2013; Hinduja y Patchin, 201; Romera, Cano, García-Fernández y Ortega, 
2016). Sin embargo, pocos estudios han explorado dicha influencia en los diferentes roles identificados en este fenómeno y con escolares de primaria.

El objetivo de este artículo es analizar cómo los factores personales y de contexto de los iguales contribuyen a la formación de los roles de no participación, espectadores, víctimas, agresores y agresores victimizados identificados en el acoso cibernético (Del Rey et al., 2015). Usando el modelo ecológico como guía, nos centraremos en los diferentes perfiles sociales señalados. Partimos de la hipótesis de que las variables de contexto de los iguales describirán mejor los diferentes comportamientos de los escolares en cyberbullying.

\section{Método}

\section{Participantes}

La población de referencia del estudio ha sido el conjunto de alumnos y alumnas que cursan el tercer ciclo de la educación primaria en Andalucía. El error muestral, teniendo en cuenta la representatividad las condiciones posibles fue de $\pm 2.5 \%$.

La muestra estuvo conformada por 1278 estudiantes (52.3\% niños y $47.7 \%$ niñas) de los últimos cursos de Educación Primaria $\left(5^{\circ}\right.$ y $\left.6^{\circ}\right)$ de la Comunidad Autónoma de Andalucía, con edades comprendidas entre los 10 y 14 años $(M=11.11 ; D T=0.75)$. El $49.4 \%$ estaba en $5^{\circ}$ de primaria y el $50.6 \%$ se encontraba en el $6^{\circ}$ curso.

Se realizó un muestreo probabilístico aleatorio estratificado por conglomerados, estableciendo estratos en función de las provincias ( 8 provincias). Los participantes pertenecían a 38 centros de primaria públicos, privados y concertados.

\section{Instrumentos}

Se utilizaron preguntas de carácter sociométrico (género, edad y curso).

Para identificar los roles se seleccionaron dos ítems de opción múltiple (nunca, pocas veces, alrededor de una vez por semana y varias veces por semana) referidos al tipo y la frecuencia de implicación en cyberbullying: Cibervictimización (¿cuántas veces te han intimidado, rechazado o maltratado a través de internet/ móvil en los últimos tres meses?); y Ciberagresión (¿has intimidado, rechazo o maltratado a alguien, a través de internet/ móvil, en los últimos tres meses?). Los ítems presentaron unos índices aceptables de asimetría y de curtosis (asimetría $=1.73$, curtosis $=1.96)$.

Para medir los factores de contexto de los iguales se utilizaron tres dimensiones de la Escala de Convivencia Escolar (ECE) (Del Rey, Casas y Ortega-Ruiz, 2017): Ajuste social con los iguales: 9 ítems $(\alpha=.79)$. Ej: Me uno a las actividades que realizan los demás; Ajuste a las normas sociales: 5 ítems $(\alpha=.87)$. Ej: Cumplo las normas; e Indisciplina y disruptividad: 4 ítems (muestra $\alpha=.70$ ). Ej: Solo cumplo las normas que me convienen.

El rendimiento académico fue medido con la pregunta “¿cómo te van los estudios?", de opción múltiple con cuatro opciones de respuesta (saco buenas notas, voy aprobando todas, apruebo pero me quedan algunas y suspendo casi todas o todas).

Por último, se seleccionó la Escala sobre autoestima de Rosenberg (1965), compuesta de 10 ítems, unos formulados en negativo y otros en positivo, que describen la confianza frente a la desconfianza, el pesimismo y el fatalismo que los escolares depositan en sí mismos. 
Las dos dimensiones que valora esta escala se evalúan mediante ítems tipo Likert de 4 opciones de respuesta que indican el grado de acuerdo o desacuerdo con las afirmaciones que se presentan siendo 1 "totalmente de acuerdo" y 4 "totalmente de acuerdo". Estas dimensiones distinguen entre autoestima positiva conformada por seis ítems $(\alpha=.716)$, y otra la autoestima negativa compuesta por cuatro ítems $(\alpha=$.647) (García-Fernández, Romera y Ortega, 2015).

\section{Procedimiento}

La selección y el contacto formal con los centros se realizó mediante un documento formal donde se indicaba la naturaleza, la finalidad y los objetivos de la actividad. Una vez aceptada la participación, miembros del equipo de investigación e investigadores formados para ello, se trasladaron a los centros educativos con el fin de administrar los cuestionarios teniendo en cuenta la disponibilidad horaria y el número de aulas. La recogida de datos fue realizada por un número determinado de encuestadores sin causar demasiadas perturbaciones a la rutina diaria de cada centro.

Para garantizar en la medida de lo posible la correcta cumplimentación del cuestionario se enfatizó en la naturaleza voluntaria y anónima del mismo. La administración de los instrumentos se hizo de manera colectiva en las respectivas aulas, sin la presencia de docentes, en una única sesión de 50 minutos.

\section{Análisis de Datos}

Para configurar los roles de implicación en cyberbullying -víctimas, agresores, agresores victimizados, no implicados y espectadores-, se utilizó el modelo teórico presentado por Del Rey y colaboradores (2015).

Para el estudio descriptivo se utilizó el análisis $\chi^{2}$ para las variables género y curso académico, para cada uno de los roles. Para el resto de variables, se realizó un análisis de la varianza (ANOVA). Para el estudio de las diferencias de medias se utilizaron las pruebas posthoc de Games Howell o Tukey dependiendo de la homogeneidad de las varianzas. Siguiendo las recomendaciones de Pedro Morales (2011) se realizó un cálculo complementario a los análisis de contrastes de medias (ANOVA), para considerar el tamaño del efecto de la muestra. Para ello, se usó el coeficiente eta cuadrado $\left(\eta^{2}\right)$ que nos indica el porcentaje de variabilidad total de la variable dependiente que se explica por el factor (Morales, 2011).

Para el tratamiento de los datos se utilizó el programa estadístico SPSS y AMOS en su versión 20.0.

\section{Resultados}

\section{Análisis descriptivos para cyberbullying}

Los análisis descriptivos de la muestra indican que población escolar, de $5^{\circ}$ y $6^{\circ}$ de Primaria, de Andalucía está implicada con altos porcentajes en problemas de cyberbullying. Si consideramos los roles más activos de la dinámica (víctimas, agresores y agresores victimizados) los datos presentan una prevalencia de $31.2 \%$ siendo los índices de participación los siguientes: no implicados (68.9\%), espectadores (12.9\%), víctimas $(9.3 \%)$, agresor $(5.5 \%)$ y agresor victimizado $(3.4 \%)$. 


\section{Relaciones con la implicación en cyberbullying}

Los análisis de Chi-cuadrado mostraron diferencias estadísticamente significativas en todas las variables estudiadas entre los distintos roles de implicación de cyberbullying. Los valores ofrecidos en función del género por los coeficientes de contingencia calculados indicaron una fuerte independencia al ser cercano a $0, \chi^{2}(4,1187)=18.547, p<.01 ; C=.124$, describiendo a los chicos en los roles de agresor victimizado y de agresor; y a las chicas en el de no implicación (ver tabla 1). Sin embargo, no se evidencian diferencias estadísticamente significativas respecto del curso y el tipo de implicación, aunque la media de implicación en $6^{\circ}$ de primaria es superior a la del $5^{\circ}$ curso (14.03\% frente a $\left.17.12 \%\right)$ (ver tabla 2$)$.

Tabla 1. Porcentajes de escolares según el género

\begin{tabular}{llccc}
\hline & & \multicolumn{2}{c}{ Sexo } & \multirow{2}{*}{ Total } \\
\hline & Hombre & Mujer & \\
\hline \multirow{3}{*}{ Perfil Completo de } & Espectadores & $34.7 \%$ & $34.2 \%^{*}$ & $68.9 \%$ \\
Ciberbullying & Víctimas & $7.0 \%$ & $5.9 \%$ & $12.9 \%$ \\
& Agresores & $4.3 \%$ & $5 \%$ & $9.3 \%$ \\
Total & Agresores victimizados & $3.9 \% *$ & $1.6 \%$ & $5.5 \%$ \\
& & $2.5 \% *$ & $0.9 \%$ & $3.4 \%$ \\
& & $52.4 \%$ & $47.6 \%$ & $100.0 \%$ \\
\hline
\end{tabular}

*Residuos tipificados corregidos $\geq|1.96|$

Tabla 2. Porcentajes de escolares según el curso

\begin{tabular}{llccc}
\hline & & \multicolumn{2}{c}{ curso } & \multirow{2}{*}{ Total } \\
\hline \multirow{3}{*}{ Perfil Completo de } & $5^{\text {o }}$ Primaria & $6^{\circ}$ Primaria & \\
Ciberbullying & No Implicado & $34.8 \%$ & $34.1 \%$ & $68.9 \%$ \\
& Espectadores & $5.7 \%$ & $7.3 \%$ & $12.9 \%$ \\
Total & Víctimas & $3.8 \%$ & $5.5 \%$ & $9.3 \%$ \\
& Agresores & $3.0 \%$ & $2.5 \%$ & $5.5 \%$ \\
& Agresores victimizados & $1.5 \%$ & $1.9 \%$ & $3.4 \%$ \\
\hline
\end{tabular}

*Residuos tipificados corregidos $\geq|1.96|$

Para el resto de variables evaluadas los estadísticos obtenidos para cada uno de los factores muestran que las medias más elevadas se encuentran en las variables ajuste social con los iguales, ajuste a las normas sociales y autoestima negativa. Y, las medias más bajas se anotan en la variable indisciplina y disruptividad (ver tabla 3). En general, los escolares andaluces estén o no implicados en el fenómeno cyberbullying, se describen como personas ajustadas a su contexto.

En el ajuste social entre iguales, las diferencias de medias entre los grupos indican que los no implicados presentan medias más elevadas con respecto a los espectadores, víctimas y agresores.

Por otro lado se observan diferencias estadísticamente significativas en función del ajuste a las normas sociales, siendo que el grupo de los no implicados presenta medias más altas que las víctimas, agresores y agresores victimizados y el grupo de los espectadores que el de los agresores y agresores victimizados. Además, las víctimas también muestran medias más elevadas que los agresores. En lo que respecta a la indisciplina y disruptividad se observa un fenómeno parecido pero de manera inversa al ajuste social a las normas. Así, los no implicados 
tienen las medias más bajas en desajuste que las víctimas, agresores y agresores victimizados y los espectadores y las víctimas tienen medias más bajas con respecto a los agresores.

Para la autoestima negativa, se observa que los no implicados y los espectadores tienen medias más bajas que las víctimas. Sin embargo, a diferencia de lo que ocurre en otras variables similares donde se observan los mismos efectos pero inversamente, la autoestima positiva no indicó diferencias de medias en los distintos grupos.

Por último, se observan diferencias estadísticamente significativas en función del rendimiento académico, siendo el grupo de escolares de no implicados y de los agresores los que presentan diferencias de medias. El análisis indica que los no implicados obtienen mejores resultados académicos que los implicados en el rol de agresor.

Tabla 3. Roles en Cyberbullying según las variables personales e interpersonales

\begin{tabular}{|c|c|c|c|c|c|c|c|c|c|}
\hline & Roles del Cyberbullying & $\mathbf{N}$ & $\mathbf{M}$ & D.T. & E. T & $\mathbf{F}$ & Sig. & $\begin{array}{c}\text { Games-Howell } \\
\text { /Tukey }\end{array}$ & $\eta^{2}$ \\
\hline \multirow{6}{*}{$\begin{array}{l}\text { Ajuste social } \\
\text { con los } \\
\text { iguales }\end{array}$} & No Implicado & 707 & 3.037 & .744 & .027 & \multirow{6}{*}{9.279} & .000 & $1^{\mathrm{o}} \neq 2^{\mathrm{o}} .3^{\mathrm{o}} .4^{\mathrm{o}}$ & \multirow{6}{*}{.909} \\
\hline & Espectadores & 134 & 2.827 & .814 & .070 & & & $2^{\circ} \neq 1^{\circ}$ & \\
\hline & Víctimas & 94 & 2.634 & .938 & .096 & & & $3^{\circ} \neq 1^{\circ}$ & \\
\hline & Agresores & 57 & 2.666 & .900 & .119 & & & & \\
\hline & Agresores Victmizados & 32 & 2.738 & .740 & .130 & & & & \\
\hline & Total & 1024 & 2.942 & .794 & .024 & & & & \\
\hline \multirow{6}{*}{$\begin{array}{l}\text { Ajuste a las } \\
\text { normas } \\
\text { sociales }\end{array}$} & No Implicado & 741 & 3.451 & .556 & .020 & \multirow{6}{*}{30.061} & .000 & $1^{\circ} \neq 3^{\circ} .4^{\circ} \cdot 5^{\circ}$ & \multirow{6}{*}{.477} \\
\hline & Espectadores & 141 & 3.333 & .658 & .055 & & & $2^{\circ} \neq 4^{\circ} .5^{\circ}$ & \\
\hline & Víctimas & 98 & 3.139 & .689 & .069 & & & $3^{\circ} \neq 1^{\circ} .4^{\circ}$ & \\
\hline & Agresores & 63 & 2.794 & .793 & .099 & & & $4^{\mathrm{o}} \neq 1^{\mathrm{o}} \cdot 2^{\mathrm{o}} \cdot 3^{\mathrm{o}}$ & \\
\hline & Agresores Victmizados & 39 & 2.771 & .780 & .124 & & & $5^{\circ} \neq 1^{\circ} .2^{\circ}$ & \\
\hline & Total & 1082 & 3.345 & .639 & .019 & & & & \\
\hline \multirow{6}{*}{$\begin{array}{l}\text { Indisciplina y } \\
\text { disruptividad }\end{array}$} & No Implicado & 753 & .725 & .653 & .023 & \multirow{6}{*}{24.998} & .000 & $1^{\circ} \neq 3^{\circ} .4^{\circ} .5^{\circ}$ & \multirow{6}{*}{.699} \\
\hline & Espectadores & 140 & .915 & .863 & .072 & & & $2^{\circ} \neq 4^{\circ}$ & \\
\hline & Víctimas & 101 & 1.070 & .716 & .071 & & & $3^{\circ} \neq 1^{\circ} .4^{\mathrm{o}}$ & \\
\hline & Agresores & 63 & 1.468 & .828 & .104 & & & $4^{\mathrm{o}} \neq 1^{\mathrm{o}} \cdot 2^{\mathrm{o}} \cdot 3^{\mathrm{o}}$ & \\
\hline & Agresores Victmizados & 37 & 1.335 & .858 & .141 & & & $5^{\circ} \neq 1^{\circ}$ & \\
\hline & Total & 1094 & .845 & .737 & .022 & & & & \\
\hline \multirow{6}{*}{$\begin{array}{c}\text { Autoestima } \\
\text { Positiva }\end{array}$} & No Implicado & 750 & 3.395 & .496 & .018 & \multirow{6}{*}{4.097} & .003 & & \multirow{6}{*}{.938} \\
\hline & Espectadores & 141 & 3.355 & .550 & .046 & & & & \\
\hline & Víctimas & 104 & 3.273 & .503 & .049 & & & & \\
\hline & Agresores & 57 & 3.218 & .566 & .075 & & & & \\
\hline & Agresores Victmizados & 38 & 3.162 & .687 & .111 & & & & \\
\hline & Total & 1090 & 3.361 & .518 & .015 & & & & \\
\hline \multirow{6}{*}{$\begin{array}{l}\text { Autoestima } \\
\text { Negativa }\end{array}$} & No Implicado & 749 & 1.845 & .652 & .023 & \multirow{6}{*}{4.958} & .000 & $1^{\circ} \neq 3^{\circ}$ & \multirow{6}{*}{.780} \\
\hline & Espectadores & 140 & 1.941 & .619 & .052 & & & $2^{\circ} \neq 3^{\circ}$ & \\
\hline & Víctimas & 104 & 2.256 & .738 & .072 & & & $3^{\mathrm{o}} \neq 1^{\mathrm{o}} \cdot 2^{\mathrm{o}}$ & \\
\hline & Agresores & 56 & 2.071 & .738 & .098 & & & & \\
\hline & Agresores Victmizados & 38 & 2.179 & .760 & .123 & & & & \\
\hline & Total & 1087 & 1.920 & .678 & .020 & & & & \\
\hline \multirow{6}{*}{$\begin{array}{l}\text { Rendimiento } \\
\text { Académico }\end{array}$} & No Implicado & 757 & 1.63 & .849 & .031 & \multirow{6}{*}{3.062} & .003 & $1^{\circ} \neq 4^{\circ}$ & \multirow{6}{*}{.615} \\
\hline & Espectadores & 148 & 1.74 & .906 & .074 & & & & \\
\hline & Víctimas & 103 & 1.81 & .961 & .095 & & & & \\
\hline & Agresores & 62 & 2.02 & .932 & .118 & & & $4^{\circ} \neq 1^{\circ}$ & \\
\hline & Agresores Victmizados & 38 & 1.84 & .945 & .153 & & & & \\
\hline & Total & 1108 & 1.69 & .881 & .026 & & & & \\
\hline
\end{tabular}

$* 1^{\mathrm{o}}=$ No Implicados. $2^{\circ}=$ Espectadores. $3^{\circ}=$ Víctimas. $4^{\mathrm{o}}=$ Agresores. $5^{\mathrm{o}}=$ Agresores Victimizados 


\section{Discusion y conclusiones}

El presente estudio analizó la asociación entre la implicación de escolares de primaria en cyberbullying y variables personales y del contexto social. En cuanto a nuestro objetivo, los resultados confirmaron que el acoso cibernético está presente en las escuelas primarias y mostraron que los factores personales y de contexto de los iguales pueden ser distinguidos como variables descriptivas entre los estudiantes.

La influencia de las variables personales fue clara. Se observaron diferencias de género. En particular, los niños estaban más implicados que las niñas en los roles de agresión, lo que también es consistente con Livingstone, Haddon, Görzig y Ólafsson, (2011). Sin embargo, el curso académico no produjo ninguna diferencia significativa, por lo que aunque algunos estudios previos habían apuntado la existencia de escolares implicados en la dinámica en función del curso, lo cierto es que habían enmarcado en los años de la secundaria con una mayor incidencia para el ciberacoso (Garaigordobil, 2011; Tokunaga, 2010).

El estudio de la relación entre la implicación en cyberbullying y la autoestima muestra que existe un vínculo entre estar implicado como víctima y tener una autoestima negativa, diferencia que es observada en comparación a los roles de no implicación o al de los espectadores. Estos resultados se apoyan en conclusiones de estudios anteriores según los cuales las víctimas tendrían una menor autoestima que aquellos que no han sufrido episodios de cyberbullying (Cénat et al., 2014; Tipandjan y Suresh, 2012; Tokunaga, 2010). Contrariamente a los resultados de algunos estudios (Tipandjan y Suresh, 2012; Tokunaga, 2010), los datos no ofrecen una correspondencia entre agresores y agresores-victimizados con la autoestima. Ello, puede explicarse por la distancia física que las nuevas tecnologías permiten, siendo que el agresor pudiera establecer una distancia emocional con las víctimas y las consecuencias sociales y psicológicas de las mismas.

Las investigaciones sobre el rendimiento académico han mostrado que los implicados en problemas de ciberacoso presentan niveles bajos en este factor ( $\mathrm{Li}$, Smith y Cross, 2012; Tokunaga, 2010). Nuestros resultados muestran que los ciberagresores presentan un bajo rendimiento académico en relación a los no implicados, en coherencia con los estudios previos (Avilés, 2010). Esta relación puede ser explicada en base a la poca motivación por las tareas académicas o la poca capacidad reflexiva que los agresores podrían tener. En las víctimas no se ha observado diferencias en función del rendimiento académico, a diferencia de lo encontrado en otros estudios (Kowalski, Limber y Agatston, 2010), tal vez porque el impacto académico en las víctimas dependa de otros factores como la frecuencia y la gravedad de la situación.

Respecto de las variables de carácter contextual, en el ajuste social entre iguales se han observado medias más altas para el grupo de los no implicados con respecto a los espectadores, víctimas y agresores, siguiendo la línea de los estudios previos que han señalado dicha falta de apoyo de los iguales a aquellos escolares implicados (Calvete et al., 2010; Hanish y Guerra, 2000; London et al., 2007). Esto hace suponer que el tipo de estrategias que utilizan los agresores para establecer relaciones los convierten en escolares con bajo nivel de aceptación. En cambio, el escaso nivel de ajuste social de las cibervíctimas podría explicarse en base a su debilidad y vulnerabilidad, lo que implica cierta dependencia con el acoso cara a cara, tal y como reconocen algunos en estudios en los que se señalan que los niños rechazados experimentan patrones estables de victimización (Veenstra, Lindenberg, Henrich y Meyers, 2010). 
Por último, el ajuste a las normas sociales del contexto ha mostrado que los no implicados estarán más ajustados al contexto normativo y presentan menos comportamientos de conflictividad y disruptividad, lo que hace suponer que a pesar de que el cyberbullying pueda darse fuera del espacio y del contexto escolar la influencia de las normas de aula es fundamental (Hinduja y Patchin, 2013; Shariff y Hoff 2007). Además, las cibervíctimas han mostrado un mayor ajuste a las normas del contexto y una menor implicación en comportamientos disruptivos que los ciberagresores, lo que podría explicar el rol que desarrollan pero también el rechazo social que sus conductas provocan entre sus iguales.

Las conclusiones de este estudio apuntan que tanto los factores personales y de contexto de los iguales explican la participación en cyberbullying, siendo quizás las variables del contexto social las que mejor describen las conductas de ciberagresión y cibervictimización (Feslt y Quandt, 2013; Hinduja y Patchin, 2013; Romera, Cano, García-Fernández, y Ortega, 2016), tal vez debido al hecho de que no podemos entender el fenómeno independientemente del contexto social en que se genera.

Estos resultados deben ser interpretados considerando una serie de limitaciones. Aunque el tamaño de la muestra es bastante amplio debemos ser cautos a la hora de generalizar los resultados. Ampliar el rango de edad de los escolares participantes permitirá llegar a conclusiones más ajustadas a esta realidad social. La utilización del autoinforme como método de medición de las variables también supone una limitación, que podría incurrir en cierta deseabilidad. A pesar de ello, este estudio tiene implicaciones prácticas que permitirán establecer programas de intervención y prevención basados en las características personales y de contexto que mueven a los implicados en esta dinámica. Como futuras líneas de investigación se plantea el estudio del cyberbullying con carácter longitudinal en el que se amplíe el rango de edad en primaria, y se incluya la medición de otros factores personales y de contexto de los iguales como el tipo de personalidad y las conductas de grupo ante las situaciones de ciberacoso.

\section{Agradecimientos}

Este trabajo se ha llevado a cabo dentro del proyecto "Estudio de la Competencia para la Gestión dela Vida Social y su Estabilidad en Estudiantes de Primaria y Secundaria de Andalucía", financiado por el Centro de Estudios Andaluces (PRY040/14). Y del proyecto "Sexting, ciberbullying y riesgos emergentes en la red: claves para su comprensión y respuesta educativa (EDU2013-44627-P)", financiado por el Plan Nacional I +D".

\section{Referencias}

Álvarez García, D., Núñez Pérez, J. C., Álvarez Pérez, L., Dobarro González, A., Rodríguez Pérez, C., y González Castro, P. (2011). Violencia a través de las tecnologías de la información y la comunicación en estudiantes de secundaria. Anales de Psicología, 27 (1), 221-231.

Avilés, J. M. (2009). Ciberbullying: Diferencias entre el alumnado de secundaria. Boletín de Psicología, 96, 79-96.

Avilés, J. M. (2010). Éxito escolar y ciberbullying. Boletín de Psicología, 98, 73-85. 
Brewer, G., y Kerslake, J. (2015). Cyberbullying, self-esteem, empathy and loneliness. Computers in Human Behavior, 48, 255-260.

Bringué, X. y Sádaba, Ch. (2011). Menores y Redes sociales Colección Foro Generaciones Interactivas/Fundación telefónica. Retrieved from http://www.generacionesinteractivas.org/page id $=1678$.

Byrne, B. (2009). Structural Equation Modeling with AMOS. Basic concepts, applications, and programming. Canada: Psychology Press.

Calmaestra, J. (2011). Cyberbullying: prevalencia y características de un nuevo tipo de bullying indirecto. Córdoba: Servicio de publicaciones de la Universidad de Córdoba.

Calmaestra, J., Ortega, R., y Mora-Merchán, J. A. (2008). Las TIC y la convivencia: un estudio sobre formas de acoso en el ciberespacio. Investigación en la Escuela, 64, 93-104.

Calvete, E., Orue, I., Estévez, A., Villardón, L., y Padilla, P. (2010). Cyberbullying in adolescents: Modalities and aggressors' profile. Computers in Human Behavior, 26(5), $1128-1135$.

Cénat, J. M., Hébert, M., Blais, M., Lavoie, F., Guerrier, M., y Derivois, D. (2014). Cyberbullying, psychological distress and self-esteem among youth in Quebec schools. Journal of Affective Disorders, 169, 7-9.

DeHue, F., Bolman, C., y Vollink, T. (2008). Cyberbullying: Youngsters' Experiences And Parental Perception. CyberPsychology Behavior, 11(2), 217-222.

Del Rey, R., Casas, J. A., \& Ortega-Ruiz, R. (2017). Desarrollo y validación de la Escala de Convivencia Escolar. Universitas Psychologica, 16(1), 1-12.

Del Rey, R., Casas, J. A., Ortega-Ruiz, R., Schultze-Krumbholz, A., Scheithauer, H., Smith, P., Thompson, F., Barkoukis, V., Tsorbatzoudis H., Brighi, A., Guarini, A., Pyzalski, J., y Plichta, P. (2015). Structural validation and cross-cultural robustness of the European Cyberbullying Intervention Project Questionnaire. Computers in Human Behavior, 50, 141-147.

Erdur-Baker, Ö. y Tanrikulu, I. (2010). Psychological consequences of cyber bullying experiences among Turkish secondary school children. ProcediaSocial and Behavioral Sciences3, 2(2), 2771-2776.

Estévez, A., Villardón, L., Calvete, E., Padilla, P., y Orue, I. (2010). Adolescentes víctimas de cyberbullying: prevalencia y características. Psicología Conductual, 18(1), 73-89.

Fanti, K. A., Demetriou, A. G., y Hawa, V. V. (2012). A longitudinal study of cyberbullying: Examining risk and protective factors. European Journal of Developmental Psychology, 9, 168-181.

Festl, R., y Quandt T. (2013). Social relations and cyberbullying: The influence of individual and structural attributes on victimization and perpetration via the internet. Human Communication Research 39, 101-126.

Fite, P. J., Colder, C. R., Lochman, J. E., y Wells, K. C. (2007). Pathways from proactive and reactive aggression to substance use. Psychology of Addictive Behaviors, 21(3), 355364.

Garaigordobil, M. (2011). Prevalencia y consecuencias del cyberbullying: una revisión. International Journal of Psychology and Psychological Therapy, 11(2), 233-254.

Garaigordobil, M. (2015). Ciberbullying en adolescentes y jóvenes del País Vasco: cambios con la edad. Anales de Psicología, 31(3), 1069-1076. 
García-Fernández, C. M., Romera, E. M., y Ortega, R. (2015). Explicative factors of face-toface harassment and cyberbullying in a sample of primary students. Psicothema, 27(4), 347-353.

Hanish, L. D. y Guerra, N. G. (2000). Predictors of peer victimization among urban youth. Social Development, 9(4), 521-543.

Hinduja, S. y Patchin, J. (2013). Social influences on Cyberbullying behaviour among middle and high school students. Journal Youth and Adolescence, 42(5), 711-722.

Hinduja, S. y Patchin, J. W. (2008). Cyberbullying: An exploratory analysis of factors related to offending and victimization. Deviant Behavior, 29(2), 129-156.

INE (2012). Encuesta sobre Equipamiento y Uso de Tecnologías de la Información y Comunicación en los hogares. Madrid: INE.

Kiriakidis, S. P. y Kavoura, A. (2010). Cyberbullying: A review of the literature on harassment through the internet and other electronic means. Family and Community Health, 33(2), 82-93.

Kline, R. (2011). Principles and practice of Structural Equation Modeling. New York: Guildford Press.

Kowalski, R. M. y Limber, S. P. (2013). Psychological, physical and academic correlates of cyberbullying and traditional bullying. Journal of Adolescent Health, 53, S13-S20.

Kowalski, R. M., Limber, S. P., y Agatston, P. W. (2008). Cyber bullying: Bullying in the digital age. Blackwell Publishing Ltd.

Kowalski, R., Limber, S., y Agatston, P. (2010). Cyber Bullying: El acoso escolar en la era digital. Bilbao: Desclée de Brower.

Li, Q., Smith, P. K., y Cross, D. (2012). Research into cyberbullying: Context. In Q. Li, D. Cross, y P. K. Smith (Eds.), Cyberbullying in the global playground: Research from international perspectives (pp. 3-12). Oxford: Blackwell.

London, B., Downey, G., Bonica, C., y Paltin, I. (2007).Social causes and consequences of rejection sensitivity.Journal of Research on Adolescence, 17(3), 481-506.

Manke, B. (2005). The impact of cyberbullying. MindOH! Foundation. Recuperado de http://www.mindoh.com/docs/BM_Cyberbullying.pdf.

Mishna, F., Khoury-Kassabri, M., Schwan, K., Wiener, J., Craig, W., Beran, T., Pepler, D., y Daciuk, J. (2016). The contribution of social support to children and adolescents' selfperception: The mediating role of bullying victimization. Children and Youth Services Review, 63, 120-127.

Morales, P. (2011). Estadística aplicada a las Ciencias Sociales. El tamaño del efecto (effectsize): análisis complementarios al contraste de medias. Recuperado de, http://www.upcomillas.es/personal/peter/investigacion/Tama\%F1oDelEfecto.pdf

Navarro, R., Yubero, S., y Larrañaga, E. (2015). Psychosocial Risk Factors for Involvement in Bullying Behaviors: Empirical Comparison between Cyberbullying and Social Bullying Victims and Bullies. School Mental Health, 7, 235-248.

Olweus, D. y Limber, S. P. (2010).Bullying in school: Evaluation and dissemination of the Olweus Bullying Prevention Program. American Journal of Orthopsychiatry, 80, 120129.

Ortega, R., Calmaestra, J., y Mora-Merchán, J. A. (2008). Cyberbullying.International Journal of Psychology and Psychological Therapy, 8(2), 183-192. 
Ortega, R., Del Rey, R., y Sánchez, V. (2011). Nuevas dimensiones de la convivencia escolar y juvenil. Ciberconducta y relaciones en la Red: Ciberconvivencia. Madrid: Observatorio Estatal de la Convivencia Escolar. Informe interno.

Patchin, J. W. y Hinduja, S. (2006). Bullies move beyond the Schoolyard: A preliminary look at cyberbullying. Youth violence and juvenile justice, 4(2), 148-169.

Rivers, I. y Noret, N. (2010). 'I h8 u': Findings from a five-year study of text and email bullying. British Educational Research Journal, 36, 643-671.

Romera Félix, E. M., Cano, J. J., García-Fernández, C., y Ortega Ruiz, R. (2016). Cyberbullying: competencia social, motivación y relaciones entre iguales. Comunicar, $48,71-79$.

Rosenberg, M. (1965).Society and the Adolescent Self-Image. Princeton, New Jersey: Princeton University Press.

Salmivalli, C. y Pöyhönen, V. (2012). Cyberbullying in Finland. In Q. Li, D. Cross, y P.K. Smith (Eds.), Cyberbullying in the global playground: Research from international perspectives (pp. 57-72). Chichester, England: Wiley-Blackwell.

Schneider, S. K., O’Donnell, L., Stueve, A., y Coulter, R. W. S. (2012). Cyberbullying, school bullying, and psychological distress: A regional census of high school students. American Journal of Public Health, 102(1), 171-177.

Shariff, S. y Hoff, D. L, (2007). Cyber bullying: Clarifying legal boundaries for school supervision in Cyberspace. International Journal of Cyber Criminology, 1(1), 76-118.

Smith, P. K., Mahdavi, J., Carvalho, M., Fisher, S., Russell, S., y Tippett, N. (2008). Cyberbullying: Its nature and impact in secondary school pupils. Journal of Child Psychology and Psychiatry, 49, 376-385.

Steinberg, L. y Monahan, K. (2007). Age differences in resistance to peer influence.Developmental Psychology, 43(6), 1531-1543.

Sureda, J., Comas, R., Morey, M., Mut, B., Salva, F., y Oliver, M. (2009). Les TIC i elsjoves a les Illes Balears: Equipament $\mathrm{i}$ usos de les tecnologies de la informació i la comunicació per partdelsjoves de les Illes Balears d'entre 15 i 16 anys. Islas Baleares: Fundació IBIT. Recuperado de http://www.ibit.org/dades/doc/2190_ca.pdf.

Tsitsika, A., Janikian, M., Wójcik, S., Makaruk, K., Tzavela, E., Tzavara, C., Greydanus, D., Merrick, J., y Richardson, C. (2015). Cyberbullying victimization prevalence and associations with internalizing and externalizing problems among adolescents in six European countries. Computers in Human Behavior, 51, 1-7.

Tokunaga, R. S. (2010). Following you home from school: A critical review and synthesis of research on cyberbullying victimization. Computers in Human Behavior, 26, 277-287.

Varjas, K., Henrich, C. C., y Meyers, J. (2009).Urban middle school students' perceptions of bullying, cyberbullying, and school safety. Journal of School Violence, 8(2), 159-176.

Veenstra, R., Lindenberg, S., Munniksma, A., y Dijkstra, J. (2010). The complex relation between bullying, victimization, acceptance and rejection: Giving special attention to status, affect and sex differences. Child Development, 81(2), 480-486. 